\title{
Revising the definition of Alzheimer's disease: a new lexicon
}

\author{
Bruno Dubois, Howard H Feldman, Claudia Jacova, Jeffrey L Cummings, Steven T DeKosky, Pascale Barberger-Gateau, André Delacourte, \\ Giovanni Frisoni, Nick C Fox, Douglas Galasko, Serge Gauthier, Harald Hampel, Gregory A Jicha, Kenichi Meguro, John O'Brien, Florence Pasquier, \\ Philippe Robert, Martin Rossor, Steven Salloway, Marie Sarazin, Leonardo C de Souza, Yaakov Stern, Pieter JVisser, Philip Scheltens
}

\begin{abstract}
Alzheimer's disease (AD) is classically defined as a dual clinicopathological entity. The recent advances in use of reliable biomarkers of $\mathrm{AD}$ that provide in-vivo evidence of the disease has stimulated the development of new research criteria that reconceptualise the diagnosis around both a specific pattern of cognitive changes and structural/biological evidence of Alzheimer's pathology. This new diagnostic framework has stimulated debate about the definition of AD and related conditions. The potential for drugs to intercede in the pathogenic cascade of the disease adds some urgency to this debate. This paper by the International Working Group for New Research Criteria for the Diagnosis of AD aims to advance the scientific discussion by providing broader diagnostic coverage of the AD clinical spectrum and by proposing a common lexicon as a point of reference for the clinical and research communities. The cornerstone of this lexicon is to consider AD solely as a clinical and symptomatic entity that encompasses both predementia and dementia phases.
\end{abstract}

\section{Introduction}

Historically, Alzheimer's disease (AD) has been conceptualised as a "dual clinicopathological entity", which to be fully ascertained requires (1) a clinical phenotype typically centred on the presence of a progressive dementia that includes episodic memory impairment as a defining feature and involvement of other cognitive domains or skills, and (2) specific neuropathological changes that usually include intraneuronal (neurofibrillary tangles) and extracellular parenchymal lesions (senile plaques), which are often accompanied by synaptic loss and vascular amyloid deposits. ${ }^{1,2}$ Because neuropathological investigations cannot be done during life (except in very limited cases by brain biopsy), AD has evolved into a predominantly clinical entity with a probabilistic diagnosis ("probable AD"). In parallel, the term AD is used by neurobiologists and neuropathologists with reference to this specific pattern of neuropathological changes. This dichotomy in the use of AD to refer to either the clinical or the neuropathological entity is a potential source of confusion, particularly in light of repeated reports that pathological changes ("Alzheimer's pathology") can exist without the concomitant clinical manifestations of AD.,5

The incremental growth of scientific knowledge around the pathogenic events and course of AD has significantly advanced our view of the disease and its defining boundaries. In 2007, the International Working Group for New Research Criteria for the Diagnosis of AD proposed a new diagnostic framework, ${ }^{6}$ intended to move beyond the National Institute of Neurological and Communicative Disorders and Stroke-Alzheimer's Disease and Related Disorders Association (NINCDS-ADRDA) criteria. ${ }^{3}$ According to these new research criteria, the diagnosis of $\mathrm{AD}$ is made when there is both clinical evidence of the disease phenotype and in-vivo biological evidence of Alzheimer's pathology. By relying on the specific clinical and biological features of the disease, the newly proposed algorithm permits diagnosis of AD with a high level of accuracy, even at the stage of earliest clinical manifestations (prodromal stage). the proposal of a "dual clinicobiological entity" that can be diagnosed during life also raises new questions about the definition of AD. For example, this framework did not initially address the nosology of AD-related states if the defining clinicobiological duality is not present. Additionally, conditions still to be considered within the new research criteria framework include the nosological classification of clinically asymptomatic individuals who are positive for biomarkers of Alzheimer's pathology, clinically symptomatic individuals without evidence of biomarker findings, or those with atypical features (atypical AD). There are now increasingly well recognised atypical presentations that include non-amnestic focal cortical syndromes, such as progressive non-fluent aphasia, logopenic aphasia, ${ }^{8}$ and posterior cortical atrophy, ${ }^{9}$ that are confirmed neuropathologically as being AD.

The aim of this Paper is to advance the new research criteria initiative by providing a companion lexicon wherein the different entities and concepts related to AD are defined and updated. This lexicon for AD is primarily intended to serve the research community by providing a framework of the disease that covers its full spectrum, and which should be used for research protocols and clinical trials directed at early intercession in the pathogenic cascade of the disease. The potential to test disease-modifying interventions adds urgency to the need for such a shared lexicon. The secondary aim is to provide clinicians with a clear view of this evolving field in which use of biomarkers is advancing and might reach regulatory qualification and approval in the foreseeable future. These dual aims are intended to keep the research and clinical view of the disease from becoming too widely separated.

\section{Methods}

In 2007, the International Working Group published the framework for new research criteria for AD. ${ }^{6}$ Subsequently, the International Working Group has convened annual meetings to advance this initiative, including considerations of how the new research criteria might be further developed
Although successfully stimulating scientific discussion,
Published Online October 11, 2010 DOI:10.1016/S14744422(10)70223-4 See Online/Reflection and Reaction DOI:10.1016/S14744422(10)70246-5

Pierre \& Marie Curie University, Paris, Research Centre of the Institute of the Brain and Spinal Cord, Institute for Memory and Alzheimer's Disease, UMR-S975, AP-HP, Pitié-Salpêtrière Hospital Group, Paris, France (Prof B Dubois MD, M Sarazin MD, L ( de Souza MD); Division of Neurology, University of British Columbia Vancouver, BC, Canada (Prof H H Feldman MD, (Jacova PhD); Department of Neurology, Yale University, New Haven, $\mathrm{CT}$, USA (H H Feldman); Bristol-Myers Squibb Neuroscience, Wallingford, CT, USA (H H Feldman); UCLA-Easton Alzheimer's Disease Research Center, Los Angeles, CA, USA (J LCummings MD); Department of Neurology, University of Virginia, Charlottesville, VA, USA (Prof ST DeKosky MD); INSERM U897, Victor Segalen University Bordeaux 2, Bordeaux, France (P Barberger-Gateau MD); Association for the Development of Applied Neurosciences,

Faches-Thumesnil, France (A Delacourte PhD); Istituto di Ricovero e Cura a Carattere Scientifico (IRCCS) Fatebenefratelli, Brescia, Italy (Prof G Frisoni MD); Dementia Research Centre, Department of Neurodegeneration, Institute of Neurology, University College London, London, UK (Prof N C Fox MD, Prof M Rossor MD); Department of Neurosciences, University of California, San Diego, CA, USA (Prof D Galasko MD); McGill Center for Studies in Aging, Douglas Hospital, Montreal, QC, Canada

(Prof S Gauthier MD); Department of Psychiatry, Psychosomatic Medicine \& 
Psychotherapy, Goethe University, Frankfurt, Germany (Prof H Hampel MD); Alzheimer's Disease Center, University of Kentucky, Lexington, $\mathrm{KY}$, USA (G A Jicha MD); Department of Geriatric Behavioural Neurology, Tohoku University Graduate School of Medicine, Sendai, Japan (K Meguro MD); Wolfson Research Centre, Institute for Ageing and Health, Newcastle University, Newcastle upon Tyne, UK (J O'Brien DM); University of Lille Nord de France (UDSL), Equipes d'Accueil (EA) 2691, Centre Hospitalier Universitaire (CHU), Lille, France (Prof F Pasquier MD); Centre Mémoire de Ressources et de Recherche CHU de Nice, University of Nice-Sophia Antipolis (UNSA), Nice, France (Prof P Robert MD); Department of Clinical Neurosciences,

Brown University, Providence, RI, USA (Prof S Salloway MD); Cognitive Neuroscience Division, Taub Institute, Presbyterian Hospital, New York, NY, USA (Prof Y Stern PhD); Department of Psychiatry \&

Neuropsychology, Alzheimer Centre Limburg, School of Mental Health and

Neuroscience, Maastricht University Medical Centre,

Maastricht, Netherlands (P J Visser MD); and Department of Neurology and Alzheimer Center, VU University Medical Centre, Amsterdam,

Netherlands (P J Visser, Prof P Scheltens MD)

Correspondence to: Prof Bruno Dubois, Department of Neurology, Salpêtrière Hospital, 47 Boulevard de I'Hôpital, 75013 Paris, France bruno.dubois@psl.aphp.fr and validated. In July, 2008, more than 50 participants with academic or pharmaceutical experience, primarily from Europe and North America, participated in a meeting during the International Conference on Alzheimer's Disease (ICAD; Chicago, IL, USA) aimed at reviewing the ongoing validation studies and aggregating data on the new research criteria. On July 13, 2009, the International Working Group hosted a featured research symposium at the 2009 ICAD meeting (in Vienna, Austria) on how the new criteria might be implemented within research studies. Following on from the interest and discussions generated at these meetings concerning the consequences of the proposed conceptual framework, a clarification and a restatement of the definition of $\mathrm{AD}$ and related states seemed necessary, and the International Working Group recognised the timeliness of an initiative to further elaborate a new lexicon.

This paper was developed through a three-step process: (1) The leaders of the International Working Group (BD, HHF, and PS) developed the broad conceptual coverage and a comprehensive first draft that was addressed to all members of the International Working Group and to other members who have been active since the 2007 publication (GF, HH, MS, LCdS). (2) Each member of the International Working Group was asked to comment and respond to the draft; all comments were collated and the lead authors engaged in discussions within the group by use of electronic communication reconciling differing viewpoints to reach a final consensus. This process led to the broadening of several important areas, including the inclusion of mixed $\mathrm{AD}$ and a firmer elaboration of atypical AD. Finally, (3) the resulting manuscript was further circulated to all coauthors for their final validation and interest in being part of the authorship of this Position Paper.

\section{Alzheimer's disease}

Currently, clinicians use the term AD to refer to a clinical entity that typically presents with a characteristic progressive amnestic disorder with subsequent appearance of other cognitive, behavioural, and neuropsychiatric changes that impair social function and activities of daily

\begin{tabular}{|lll|}
\hline & $\begin{array}{l}\text { Pathophysiological } \\
\text { markers }\end{array}$ & $\begin{array}{l}\text { Topographical } \\
\text { markers }\end{array}$ \\
\hline Cerebrospinal fluid & Yes & No \\
$\begin{array}{l}\text { Amyloid } \beta_{42} \\
\text { Total tau, phospho-tau }\end{array}$ & Yes & No \\
PET & Yes & No \\
Amyloid tracer uptake & No & Yes \\
Fluorodeoxyglucose & & Yes \\
Structural MRI & No & \\
Medial temporal atrophy & & \\
AD=Alzheimer's disease. & & \\
\hline Table 1: Categorisation of the current, most-validated AD biomarkers
\end{tabular}

living. ${ }^{1}$ The initial presentation can also be atypical, with non-amnestic focal cortical cognitive symptoms. ${ }^{9}$ In most cases, clinicians make this diagnosis of $\mathrm{AD}$ with varying degrees of confidence on the basis of their understanding and weight of evidence for a typical or atypical phenotypic presentation. To address this diagnostic uncertainty, the 1984 NINCDS-ADRDA criteria stipulated that diagnosis of AD during life could only be "probable", whereas a "definite" diagnosis required post-mortem histopathological confirmation. ${ }^{3}$ Additionally, the diagnosis of probable $\mathrm{AD}$ could be made only when the severity threshold for dementia was reached and when other causes of dementia had been excluded by biological and neuroimaging examination. However, as noted, the term $\mathrm{AD}$ has also been used by researchers to refer to the pathological process alone. As a consequence, the original clinicopathological duality traditionally inherent in the term AD has gradually eroded.

Over the past two decades, it has become increasingly possible to identify in-vivo evidence of the specific neuropathology of AD by use of validated and diseasespecific biomarkers. ${ }^{10-12}$ This reliable identification of AD biomarkers supports a major change in the conceptualisation and diagnosis of $\mathrm{AD}$, because both clinical and in-vivo biological manifestations of the disease can now be integrated into the diagnosis. Laboratory and neuroimaging biomarkers are very highly correlated with the neuropathological lesions of AD. ${ }^{13-17}$ These biomarkers can be divided into pathophysiological and topographical markers (table 1).

Pathophysiological markers correspond to the two aetiological degenerative processes that characterise Alzheimer's pathology: the amyloidosis path to neuritic plaques and the tauopathy path to neurofibrillary tangles. ${ }^{18}$ They include CSF measures of reduced concentrations of amyloid $\beta$, increased total tau, and increased phosphotau, ${ }^{13,14,16,17,19-22}$ and amyloid PET scanning with Pittsburgh compound $\mathrm{B}(\mathrm{PiB})^{15,23,24}$ or other radioligands (florbetaben, 18F-AV-45, etc). ${ }^{25,26}$ CSF biomarkers (low amyloid $\beta$ and, even more specifically, abnormal ratio of tau to amyloid $\beta)^{27}$ are associated with very high rates of progression from amnestic mild cognitive impairment (MCI) to AD dementia, ${ }^{21,28}$ and have shown a consistently high sensitivity and specificity in predictive models. ${ }^{27-30}$ High mean cortical binding values for PiB-PET are predictive of cognitive decline and development of AD clinical signs in cognitively normal elderly individuals. ${ }^{31,32}$ In-vivo pathophysiological markers correlate very well with their respective neuropathological lesions, including CSF amyloid $\beta$ and PiB-PET with senile plaques, ${ }^{15-17,19}$ and total tau and phospho-tau with neurofibrillary tangles..$^{13,14,17,19}$

Topographical markers are used to assess the less specific and downstream brain changes that correlate with the regional distribution of Alzheimer's pathology and include medial temporal lobe atrophy ${ }^{33-37}$ and reduced glucose metabolism in temporo-parietal regions on fluorodeoxyglucose PET. ${ }^{38}$ These markers are valuable 
indicators because structural brain changes accurately map to the Braak stages of neurofibrillary tangle deposition. ${ }^{39}$ These MRI and PET topographical markers have been shown consistently to predict the development of AD dementia in MCI cohorts, ${ }^{31,40-44}$ and to correlate with disease severity. ${ }^{45,46}$

Among the pathophysiological and topographical biomarkers, some have greater specificity for Alzheimer's pathology and might provide a different likelihood of an AD diagnosis within a particular context. For example, because medial temporal lobe atrophy can occur in non-AD dementias ${ }^{47}$ or with ageing, a low CSF amyloid $\beta_{42}$ or a positive amyloid result on PET imaging might have higher specificity for the amyloidosis associated with AD than would medial temporal lobe atrophy. In 2007, at the time of publication of the new research criteria, ${ }^{6}$ there was no empirical basis for assigning weightings to pathophysiological versus topographical markers in the diagnosis of AD. Since then, understanding of the timing of pathological events has increased, with pathophysiological changes preceding the topographical changes associated with neurodegeneration..$^{48}$ The latter are more related to the emergence of cognitive symptoms, ${ }^{49,50}$ although regional hypometabolism has been shown with fluorodeoxyglucose PET in asymptomatic adults with genetic susceptibility for AD. ${ }^{51-54}$ According to this sequence of events, pathophysiological markers could have diagnostic use at all disease stages, including the preclinical stage, whereas topographical markers would be more useful closer to the time when the first cognitive symptoms are manifest.

The availability of in-vivo biomarkers and their correspondence with Alzheimer's pathology form the basis of the new research criteria, which were founded on a clinicobiological definition. ${ }^{6}$ AD is now defined in vivo with a diagnostic algorithm that begins with a characteristic pattern of episodic memory impairment and then requires supportive biomarkers that indicate the pathophysiology or the topography of Alzheimer's pathology. The presence of dementia itself-the more severe form of $\mathrm{AD}$ - is not required. The diagnosis of $\mathrm{AD}$ is made on the basis of both clinical and biological evidence, with a very high level of specificity and predictive validity. ${ }^{55}$ Within this framework, the designation of "probable" and "possible" AD is no longer meaningful because of the use of reliable biomarkers and the designation of "typical" and "atypical" AD.

The argument for defining $\mathrm{AD}$ as a clinicobiological entity with a specified clinical phenotype and in-vivo evidence of the footprint of pathological changes has the major advantage that there is no longer a reason to wait until patients have developed full-blown dementia or to exclude from diagnosis and treatment a large number of patients who lack functional disability yet express the disease. The diagnosis in turn can be uncoupled from a particular threshold of severity, and there is no longer a need to anchor the diagnosis of $\mathrm{AD}$ to a dementia syndrome as is done today. Here, it is useful to refer to
Parkinson's disease in cases in which the diagnosis does not hinge on a level of severity (eg, when the patient is bedridden), but on the presence of the earliest motor symptoms (eg, a limited resting tremor of one hand). The same should apply for $\mathrm{AD}$, whereby the presence of a distinctive episodic memory impairment pattern, ${ }^{6}$ together with biological evidence on structural MRI, ${ }^{36}$ molecular neuroimaging with $\mathrm{PET},{ }^{43}$ or CSF analysis ${ }^{29}$ identifies AD with high accuracy at a symptomatic predementia stage..$^{55}$ However, biomarkers are only supportive features in the diagnostic framework, which is anchored to a core clinical phenotype. Accordingly, AD has been identified in patients 2 years before dementia with a specificity of $92 \%$ using an episodic memory test that assesses response to cueing. ${ }^{56}$ When CSF and volumetric MRI measures are added to memory impairment, an extremely high degree of specificity has been reported. ${ }^{57-59}$ Use of the 2007 research criteria for $\mathrm{AD}^{6}$ has been reported to capture the prodromal phase of the disease, ${ }^{57,59,60}$ and supports the validity of a diagnosis of $\mathrm{AD}$ before the occurrence of full-blown dementia with functional impairment.

In summary, on the basis of this novel diagnostic approach and on the evidence accumulated to date, we propose that the term "Alzheimer's disease" should refer only to the in-vivo clinicobiological expression of the disease and that it should encompass the whole spectrum of its clinical course. Although prospective studies with post-mortem verification are needed to validate this new proposal, the diagnosis of typical AD can now be made in vivo with very high specificity, given the specificity of the hippocampal pattern of memory loss with supportive biomarkers. Accordingly, we anticipate that in the future a single in-vivo marker or, more likely, a combination of markers might be as reliable as neuropathological criteria in establishing a definite diagnosis of AD. However, to reach this point, the new clinicobiological definition needs to be compared with the traditional dual neuropathological diagnosis before establishing its validity as a new gold standard. Furthermore, post-mortem examination remains useful for the identification of comorbid conditions, alternative diagnoses (such as argyrophilic grain disease), and atypical cases. In this lexicon, we propose that within the framework provided by the new research criteria, "definite AD" should be replaced with "neuropathologically verified $A D$ ", whereby a post-mortem examination is used to verify the clinicobiological diagnosis (panel).

\section{Alzheimer's pathology}

For basic neuroscience and neuropathology researchers, $\mathrm{AD}$ refers to the pathological process that is defined by specific neuronal lesions including senile plaques and neurofibrillary tangles, and that is associated with neuronal loss, synaptic loss, and frequently with cerebral amyloid angiopathy. ${ }^{18}$ This pathological process might or might not become symptomatic during life. ${ }^{61,62}$ For research and clinical purposes, we propose to refer to the underlying pathology of the disease at the genetic, molecular, or 
cellular level as "Alzheimer's pathology". Alzheimer's pathology can be identified post mortem in the absence of any significant evidence of cognitive or behavioural

\section{Panel: A new lexicon for Alzheimer's disease}

\section{Alzheimer's disease (AD)}

This diagnostic label is now restricted to the clinical disorder that starts with the onset of the first specific clinical symptoms of the disease, and encompasses both the predementia and dementia phases. $A D$ thus refers to the whole spectrum of the clinical phase of the disease and is not restricted to the dementia syndrome. The diagnosis is now established in vivo and relies on a dual clinicobiological entity that requires the evidence of both specific memory changes and in-vivo markers of Alzheimer's pathology that can include: CSF amyloid $\beta$, total tau, and phospho-tau; retention of specific PET amyloid tracers; medial temporal lobe atrophy on MRI; and/or temporal/ parietal hypometabolism on fluorodeoxyglucose PET. The clinical phenotype can be typical or atypical. Additionally, two different stages might still be meaningful: a prodromal and a dementia phase.

\section{Prodromal AD (also called "predementia stage of $A D$ ")}

This term refers to the early symptomatic, predementia phase of $A D$ in which (1) clinical symptoms including episodic memory loss of the hippocampal type (characterised by a free recall deficit on testing not normalised with cueing) are present, but not sufficiently severe to affect instrumental activities of daily living and do not warrant a diagnosis of dementia; and in which (2) biomarker evidence from CSF or imaging is supportive of the presence of $A D$ pathological changes. This phase is now included in the new definition of $A D$. The term of prodromal $A D$ might disappear in the future if $A D$ is considered to encompass both the predementia and dementia stages.

\section{AD dementia}

This term refers to the phase of $A D$ during which cognitive symptoms are sufficiently severe to interfere with social functioning and instrumental activities of daily living, a threshold that is considered to define dementia in association with changes in episodic memory and in at least one other cognitive domain. It might still be meaningful to identify the dementia threshold for clinical trials or social/economic evaluations.

\section{Typical AD}

This term refers to the most common clinical phenotype of $A D$, which is characterised by an early significant and progressive episodic memory deficit that remains dominant in the later stages of the disease, and is followed by or associated with other cognitive impairments (executive dysfunction, language, praxis, and complex visual processing impairments) and neuropsychiatric changes. The diagnosis is further supported by one or more in-vivo positive biomarkers of Alzheimer's pathology.

\section{Atypical AD}

This term refers to the less common and well characterised clinical phenotypes of the disease that occur with Alzheimer's pathology. These clinical syndromes include primary progressive non-fluent aphasia, logopenic aphasia, frontal variant of $A D$, and posterior cortical atrophy. In the presence of one of these clinical presentations, the diagnosis of AD is supported by in-vivo evidence of amyloidosis in the brain (with retention of specific amyloid labelling radioligands) or in the CSF (with changes characteristic of Alzheimer's pathology in amyloid $\beta$, tau, and phospho-tau concentrations).

\section{Mixed AD}

This term refers to patients who fully fulfil the diagnostic criteria for typical $A D$ and additionally present with clinical and brain imaging/biological evidence of other comorbid disorders such as cerebrovascular disease or Lewy body disease.

(Continues on next page) changes in vivo. ${ }^{4}$ Because of lack of a clear understanding of the relation between the neuropathological pattern of the disease and its clinical occurrence, we believe that it could be confusing to continue to use the term of $\mathrm{AD}$ for both conditions, and we propose to distinguish between AD expressed clinically and Alzheimer's pathology, which might or might not be clinically manifest. Neuropathologically, the National Institute on Aging-Reagan criteria of "intermediate likelihood" can occur at a frequency of $10-36 \%$ in cognitively normal individuals followed to autopsy. ${ }^{4}$ We thus propose to clearly separate the clinical expression of the disease (AD) from the underlying pathology (Alzheimer's pathology; panel).

\section{Preclinical states of Alzheimer's disease}

There is growing interest in the long preclinical phase of AD. ${ }^{4,3149,62,63}$ Within this lexicon, we distinguish two preclinical states of $\mathrm{AD}$ in which individuals are free of cognitive/behavioural symptoms, yet have either biomarker evidence of Alzheimer's pathology or a monogenic form of $\mathrm{AD}$. We propose the term "asymptomatic at risk for AD" for individuals with biomarker evidence of Alzheimer's pathology, whereas "presymptomatic AD" is designated for carriers of monogenic forms of $\mathrm{AD}$ in whom the disease will invariably occur.

\section{Asymptomatic at-risk state for $A D$}

With the development of amyloid PET imaging, fibrillar amyloid (one of the neuropathological hallmarks of the disease) can be seen in a substantial proportion of ageing individuals who are still cognitively normal and free of symptoms ("asymptomatic at risk for AD"). 12,61 Positive PiB-PET scans are reported in 10-30\% of older cognitively normal controls. ${ }^{10,12,49,61,64}$ Similarly, decreased CSF amyloid $\beta_{42}$ has been shown in asymptomatic normally functioning individuals. ${ }^{12,22}$ These individuals can be defined as healthy, and might or might not later fulfil clinical AD diagnostic criteria. This evolution might depend on individual susceptibility, including genetic factors (eg, APOE genotype), ${ }_{1}$ other risk or protective factors (eg, vascular factors, diet, etc), and comorbidities (eg, diabetes). Recent data from follow-up studies indicate that healthy individuals with abnormal CSF biomarkers and PiB-PET positive changes develop AD at an increased rate. $27,31,32,65-67$ Larger follow-up studies of asymptomatic individuals with positive amyloid- $\beta$ and tau biomarkers will clarify their risk for AD. However, because individuals who have been documented to be cognitively normal do have evidence of senile plaques and neurofibrillary tangles on autopsy, ${ }^{4}$ some of the people who have positive PET amyloid tracers will clearly not go on to develop cognitive impairment during their lifetimes. At this point, as we cannot determine this group who will remain asymptomatic, we will have to assume that they will be at high risk of developing clinical symptoms, and would be candidates for preventive therapies, depending on the risk, expense, and side-effects of such therapies, and 
depending on the progress in determining which ADbiomarker-positive healthy elderly people are unlikely to develop clinical symptoms.

The discovery of specific biomarkers of the disease has created new opportunities for a progressively earlier recognition of the disease. The timing of pathological events, as currently understood, ${ }^{49}$ confers primary importance in this context to in-vivo markers of amyloidosis. The borders between clinical AD and the preclinical states of the disease might shift in the future given continuing progress in the identification of early cognitive/behavioural changes (eg, decreased verbal fluency, attention deficits, or executive dysfunction). Recent data suggest that it might be possible to document at an earlier stage the clinical onset of AD on the basis of a precise clinical and/or neuropsychological investigation. ${ }^{68,69}$ However, it is likely that the earlier the diagnosis is made, the less accurate it will be if no specific pattern of memory or cognitive impairment has yet developed. For example, patients who present with subjective isolated memory complaints in the absence of objective evidence of a specific memory deficit, but with biomarker evidence positive for brain amyloidosis, could be wrongly diagnosed as having $\mathrm{AD} .^{22}$ For early disease recognition, there is a clear need to characterise the clinical phenotype that is highly specific for AD. This was the goal of the 2007 research diagnostic criteria. ${ }^{6}$ In the absence of specific AD cognitive signs, we suggest that AD should not be diagnosed, even if there is biological evidence of pathology, but rather the possibility of categorising these patients as having MCI should be considered.

For the asymptomatic state, because there is no empirical basis yet to determine which biological changes will be definitely associated with the further development of the clinical symptoms of $\mathrm{AD},{ }^{49,61}$ we recommend that this state should be referred to as "asymptomatic at-risk state for AD".

\section{Presymptomatic AD}

This term is applied to individuals who will develop AD because they carry a fully penetrant genetic mutation, but who are still free of symptoms of AD. At present, the only case that fulfils this requirement is that of a carrier of a familial genetic autosomal dominant mutation causative of AD. These individuals are generally referred to as having familial AD. To avoid any confusion with non-genetic familial aggregation of the disease, we now propose to introduce the term "monogenic AD" to characterise these individuals with known autosomal dominant or other well characterised single-gene mutations.

Cohorts of patients with preclinical AD are likely to attract growing interest for intervention trials to prevent $\mathrm{AD}^{70}$ It is possible that in the future, disease-modifying treatments could be directed at clinically asymptomatic individuals with biological evidence of Alzheimer's pathology who are at highest risk of further developing clinical AD..$^{71}$ The presymptomatic population is attractive
(Continued from previous page)

\section{Preclinical states of $A D$ (including both "asymptomatic at-risk state for $A D$ " and} "presymptomatic $A D$ ")

These terms refer to the long asymptomatic stage between the earliest pathogenic events/ brain lesions of $A D$ and the first appearance of specific cognitive changes. Traditionally, a preclinical or asymptomatic phase was recognised post mortem by evidence of histological changes typical of Alzheimer's pathology in individuals considered as cognitively normal before death. Today, two preclinical states can be isolated in vivo:

- Asymptomatic at-risk state for $A D$-this state can be identified in vivo by evidence of amyloidosis in the brain (with retention of specific PET amyloid tracers) or in the CSF (with changes in amyloid $\beta$, tau, and phospho-tau concentrations). In the absence of knowledge about the value of these biological changes to predict the further development of the disease, the asymptomatic phase of AD should still be referred to as an "at-risk state for AD".

- Presymptomatic $A D$-this state applies to individuals who will develop $A D$. This can be ascertained only in families that are affected by rare autosomal dominant monogenic $A D$ mutations (monogenic $A D$ )

\section{Alzheimer's pathology}

This term refers to the underlying neurobiological changes responsible for $A D$ that span the earliest pathogenic events in the brain and that include specific neuronal brain lesions (senile neuritic plaques and neurofibrillary tangles), synaptic loss, and vascular amyloid deposits within the cerebral cortex. This term can be applied irrespective of the existence of clinical manifestation.

\section{Mild cognitive impairment $(\mathrm{MCl})$}

This term applies to individuals with measurable $\mathrm{MCl}$ in the absence of a significant effect on instrumental activities of daily living. This diagnostic label is applied if there is no disease to which $\mathrm{MCl}$ can be attributed. It remains a term of exclusion for individuals who are suspected to have but do not meet the proposed new research criteria for $A D$, in that they deviate from the clinicobiological phenotype of prodromal AD because they have memory symptoms that are not characteristic of $A D$ or because they are biomarker negative.

given the certainty of $\mathrm{AD}$, but this group is very rare $(0 \cdot 3 \%$ of AD cases). Asymptomatic individuals "at risk for AD" are more common, but this population has a lower risk of developing AD. However, the probability of subsequent development of clinical AD can be substantially increased by recruiting asymptomatic individuals with a combination of risk factors (eg, older age, APOE $\varepsilon 4$ genotype, increased retention of amyloid radioligand in the brain, and evidence of age-related decreased volume of the hippocampus), ${ }^{36,40-43}$ but without achieving certainty that AD will develop or by when. Because of this uncertainty and for ethical reasons, we emphasise that reference to preclinical AD should be avoided. Accordingly, at the diagnostic or clinical level, any (clinically asymptomatic) individual should be described as being "at risk for $\mathrm{AD}$ " or having "asymptomatic amyloidopathy", but should not be defined as having preclinical AD (panel, table 2).

\section{Prodromal (predementia) Alzheimer's disease}

The term "prodromal AD" was introduced recently. ${ }^{72,73}$ It characterises clinically affected patients who do not yet have dementia (predementia) and who are diagnosed to have $\mathrm{AD}$ on the basis of their clinical presentation and 


\begin{tabular}{|c|c|c|c|c|}
\hline & AD diagnosis & $\begin{array}{l}\text { Presence of impairment on } \\
\text { specified memory tests }\end{array}$ & $\begin{array}{l}\text { Evidence of } \\
\text { biomarkers in vivo }\end{array}$ & Additional requirements \\
\hline Typical AD & Yes & Required & Required & None \\
\hline Atypical AD & Yes & Not required & Required & Specific clinical presentation \\
\hline Prodromal AD & Yes & Required & Required & Absence of dementia \\
\hline AD dementia & Yes & Required & Required & Presence of dementia \\
\hline Mixed AD & Yes & Required & Required & Evidence of comorbid disorders \\
\hline \multicolumn{5}{|l|}{ Preclinical AD } \\
\hline Asymptomatic at risk for $A D$ & No & Not present & Required & Absence of symptoms of $A D$ \\
\hline Presymptomatic AD & No & Not present & Not required & $\begin{array}{l}\text { Absence of symptoms of } A D \text { and presence of } \\
\text { monogenic } A D \text { mutation }\end{array}$ \\
\hline Mild cognitive impairment & No & Not required & Not required & Absence of symptoms or biomarkers specific for $A D$ \\
\hline$A D=A l z h e i m e r ' s$ disease. & & & & \\
\hline
\end{tabular}

supportive evidence of Alzheimer's pathology from biomarkers. Prodromal AD should not be confused with preclinical AD. Prodromal AD describes a symptomatic disease phase, no matter how early, whereas preclinical $\mathrm{AD}$ describes the preceding asymptomatic state. The new definition of $\mathrm{AD}$ proposed in this lexicon encompasses prodromal AD. Previously, individuals with the features of prodromal $\mathrm{AD}$ were described as having $\mathrm{MCI},{ }^{74}$ with an increased risk of developing $\mathrm{AD}$, but not as having identifiable AD. The proposed conceptual shift is to consider a patient previously diagnosed as having MCI (ie, with an amnestic syndrome of the hippocampal type and with biomarker evidence positive for brain amyloidosis) to be no longer at risk for developing $\mathrm{AD}$ dementia, but to recognise that they already have AD at a prodomal stage with an inevitable progression to $\mathrm{AD}$ dementia over time. A clinical phenotype combined with biomarker evidence will now no longer be predictive of $\mathrm{AD}$ but diagnostic, in accordance with the new criteria. The diagnosis of prodromal $\mathrm{AD}$ is preferred to that of MCI, because identifying the disease responsible for the syndrome is more valuable for the patient in terms of prognosis and treatment. The term "prodromal AD" is proposed in reference to the dementia phase (panel). It might subsequently disappear if $\mathrm{AD}$ is considered universally to encompass both the predementia and dementia stages (panel).

\section{Alzheimer's disease dementia}

It might still be meaningful to identify the dementia threshold as a severity milestone in the course of the disease with foreseeable clear economic and social implications. ${ }^{75}$ The transition to dementia predictably adds a set of management issues for clinicians to address, including those related to patient autonomy such as driving, financial capacity, and those related to caregiving. Given this transitional significance, the time to Alzheimer's dementia from its prodromal stage might be a useful outcome measure to consider for the study of efficacy of disease-modifying treatments, ${ }^{71}$ although the precise time of progression to this threshold might remain difficult to establish. ${ }^{31,76}$ The dementia threshold separates clinical AD into two different symptomatic phases: the prodromal (predementia) phase and the dementia phase (panel).

\section{Typical Alzheimer's disease}

The careful studies of Braak and Braak ${ }^{7}$ and Delacourte and colleagues ${ }^{78}$ have established the typical natural history of regional brain neuropathology and lesion patterns of $\mathrm{AD}$, for which there is initial neurofibrillary involvement of the entorhinal cortex, the hippocampus, and related medial temporal structures, and which subsequently spreads to the neocortical association areas. This pathway of regional neuropathology correlates with the typical pattern of the cognitive changes of $\mathrm{AD}$ in which an amnestic syndrome of the hippocampal type occurs as an early core manifestation, ${ }^{56}$ often associated with less specific changes in executive functions, naming abilities, or attention resources. Accordingly, the new research criteria emphasised the presentation of a significant progressively worsening episodic memory deficit that remains predominant in the course of the disease (panel). They focused on this phenotype because it is the most frequent, ${ }^{1}$ and it has been associated with post-mortem Alzheimer's pathology in all examined cases. ${ }^{79}$

An amnestic syndrome of the hippocampal type is now a core feature that is essential for the diagnosis of typical AD. ${ }^{56}$ This syndrome is established on the basis of patient/informant reports of memory decline and of objective evidence of episodic memory impairment on tests that control for an effective registration of the items to be remembered and probe response to cueing as a measure of the storage abilities and associative function of the hippocampus. The amnestic core diagnostic criterion is also required in more advanced cases, in which the history of an early and significant episodic memory deficit increases the specificity of the diagnosis of clinical AD. Patients with typical AD are of interest for pharmaceutical trials because high diagnostic specificity 
is required for the development of drugs directly targeted against the pathogenic mechanisms of AD (panel).

\section{Atypical Alzheimer's disease}

There are well defined clinical phenotypic variant presentations of $\mathrm{AD}$ that do not follow the typical pattern described above. These include non-amnestic focal cortical syndromes, such as primary progressive nonfluent aphasia, logopenic aphasia, posterior cortical atrophy, ${ }^{9}$ and frontal variant $\mathrm{AD} .^{80,81}$ With the advent of biomarkers providing in-vivo confirmation of Alzheimer's pathology, it is now possible to include these clinical disorders as atypical AD if there is such biomarker support. ${ }^{55.79}$ These disorders might present with an amnestic deficit only later in the disease course.

Until recently, the AD aetiology of these disorders was under-recognised clinically and only identified at post mortem if there was a compatible pattern of histological changes. ${ }^{18,82}$ We propose to use the diagnostic label of atypical AD for well characterised clinical presentations (primary progressive aphasia, logopenic aphasia, posterior cortical atrophy, frontal variant) only if they are supported by a positive pathophysiological biomarker study of Alzheimer's pathology, because these clinical conditions could also result from other pathological processes. The term "atypical" in these cases is applied only because the specific clinical presentations are much less common than the amnestic presentation. We anticipate that these atypical clinical syndromes will become better recognised and in turn more frequent in the future with the availability of in-vivo biomarkers of Alzheimer's pathology.

Pathophysiological markers including low amyloid $\beta$ and high tau CSF concentrations and increased retention of PET amyloid tracers are anticipated to have the same use as in the diagnosis of typical AD, although this remains to be validated. Topographical markers need to be considered with regard to the specific regional anatomical distribution of pathology that characterises each syndrome. Neuroimaging markers have been found to closely reflect both the clinical syndrome and the underlying distribution of pathology ${ }^{83,84}$ Neuroimaging markers that are uniquely associated with each described variant are likely to be refined. In consideration of important genetic factors, the presence of a proven autosomal dominant mutation within the immediate family is taken as sufficient evidence to support a diagnosis of atypical AD when clinical features fall outside the typical criteria (panel).

\section{Mixed Alzheimer's disease}

Mixed AD is a diagnostic confound representing the co-occurrence of Alzheimer's pathology with other biological causes of cognitive decline, typically cerebrovascular disease or Lewy body pathology (panel). These comorbid conditions might present as overlapping clinical phenotypes of disease. Mixed pathologies are highly prevalent in elderly community-dwelling adults, and the contribution of co-occurring diseases to cognitive deterioration needs to be considered in any diagnostic scheme. ${ }^{72,74}$ Clinical diagnostic consideration for mixed AD could be supported in the setting of an otherwise typical clinical phenotype of $\mathrm{AD}$ by one of several elements, including past or recent history of stroke, presence of gait disturbances, parkinsonism, hallucinations/delusions, cognitive fluctuations, and evidence of significant levels of small vessel ischaemic changes, strategic lacunar infarcts, or large vessel infarcts on brain imaging. However, detecting these co-occurrences in a patient with multiple disorders that potentially affect cognitive performance does not equate to proof of multiple causation. Although mixed dementia has never been properly operationalised, we propose that history of stroke in the absence of imaging signs of cerebrovascular disease or imaging signs of cerebrovascular disease in the absence of clinical symptoms of stroke are not sufficient to support a diagnosis of mixed dementia due to Alzheimer's pathology and cerebrovascular disease. Accordingly, to avoid an unsupported inflation of mixed dementia cases, we recommend reserving the "mixed AD" label for cases for which both clinical features and diagnostic markers point to a mixed aetiology. For example, a typical AD phenotype with white matter changes cannot be diagnosed as mixed $\mathrm{AD}$ in the absence of motor symptoms or gait disturbances consistent with the distribution of vascular pathology.

The advances in biomarker development now allow biological evidence to be used in support of a diagnosis of mixed AD. This might improve the classification of AD patients with comorbid disorders. The caveat is that cerebrovascular disease can result in increased concentrations of CSF total tau, so this biomarker should not be used to support such a diagnosis, whereas low CSF amyloid $\beta$ or positive amyloid-ligand PET imaging are sufficient to establish such a diagnosis. In the case of AD with Lewy bodies, the situation is even more complex because low CSF amyloid $\beta$ and increased amyloid-ligand binding have been reported..$^{85-88}$ In this setting, there is a limited ability to reliably identify Lewy bodies with invivo biomarkers. The most accurate approach to the Lewy body portion of the diagnosis is through phenotypic identification that includes the presence of extrapyramidal signs, hallucinations, and cognitive fluctuations, ${ }^{89}$ with positive dopamine transporter imaging that provides evidence of presynaptic dopaminergic denervation in the striatum, ${ }^{90,91}$ consistent with the diagnosis of dementia with Lewy bodies. ${ }^{89,92}$

\section{Mild cognitive impairment}

MCI is a heterogeneous condition characterised by mild cognitive changes associated with various underlying aetiologies. ${ }^{93}$ The term MCI has therefore included some patients in the symptomatic prodromal phase of AD. Because patients with prodromal AD are now reclassified by their aetiology, they are no longer included within an MCI grouping. In the absence of any specifications of the 
memory profile and of any reference to specific biomarkers, MCI or amnestic MCI remains a syndromic classification that cannot be used for the diagnosis of prodromal AD.

Because it is possible to diagnose AD at the prodromal stage, MCI now includes individuals who do not meet criteria for prodromal AD. This term is also valuable for those without a clear diagnosable disease. Accordingly, it remains a useful clinical designation for describing individuals who have a memory deficit characteristic of prodromal AD (ie, amnestic syndrome of hippocampal type), but when biomarker evidence of Alzheimer's pathology is absent, uncertain, or testing has not been done. MCI would also apply to individuals with mild cognitive impairment or memory disorders that are not consistent with prodromal AD, although biomarker evidence is present (panel).

\section{Conclusions}

The value of these definitions is their potential application in clinical trials of disease-modifying drugs. Individuals identified as "asymptomatic at risk for AD" or "presymptomatic AD" might be enrolled in trials aimed at delaying the onset of clinical signs. Patients with prodromal AD could be included in trials of drugs targeting progression to more severe stages of $\mathrm{AD}(\mathrm{AD}$ dementia). Uniformity of definitions will assist in constructing trial populations and comparing results across trials. We acknowledge that there are different opinions on the terminology and that this lexicon is a proposal that will require revisions and updating by the scientific community.

\section{Contributors}

BD, HHF, CJ, and PS developed the concept of the new lexicon. BD and HHF wrote the paper, which was critically revised by all authors, who individually approved the final version. BD had full access to all discussions and had final responsibility for the decision to submit the paper for publication.

\section{Conflicts of interest}

BD has consulted or served on advisory boards for Bristol-Myers Squibb, Novartis, Roche, Elan, Eli Lilly, Eisai, GE Healthcare, Janssen, and Sanofi-Aventis. His institution has received grants from Novartis, Roche, Eisai, and Sanofi-Aventis. HHF has consulted or served on advisory boards and/or study steering committees for AstraZeneca, Bristol-Myers Squibb, Elan, Janssen, Merck Serono, Lundbeck, Novartis, Neuropharma, Wyeth, Noscira, Servier, Glia Scientific, Eisai, and Pfizer. He has received grants for clinical trials sponsored by Elan Pharmaceuticals and Sanofi Synthelabo and performed at the University of British Columbia (UBC). He has received travel/accommodation covered or reimbursed for CME programmes from the Academy of Health Care Education, Alpha Plus, Cadmus Media, Janssen Pharma, Lundbeck, Eisai, AstraZeneca, Pfizer, Medical Decision Point, Informed Direct PLC, Med Plan Communications, and Novartis. He has held stock in Biomarin. From Jan 1, 2009, he has been on leave from UBC and is a full-time employee of Bristol-Myers Squibb. JLC has provided consultation to Abbott, Acadia, Accera, ADAMAS, Astellas, Avanir, Bristol-Myers Squibb, CoMentis, Eisai, Elan, EnVivo, Forest, GlaxoSmithKline, Janssen, Lilly, Lundbeck, Medivation, Merck, Merz, Myriad, Neuren, Neurokos, Novartis, Noven, Orion, Pfizer, Prana, reMYND, Schering Plough, Signum Bioscience, Sonexa, Takeda, Toyama, and Wyeth pharmaceutical companies, and to Bayer, MedAvante, Neurotrax, and UCB. He holds stock in ADAMAS, Prana, Sonexa, MedAvante, Neurotrax, and Neurokos. He has been a speaker/ lecturer for Eisai, Forest, Janssen, Novartis, Pfizer, Lundbeck, and Merz, owns the copyright of the Neuropsychiatric Inventory, and has provided expert witness consultation regarding olanzapine and ropinerol. STDK has collaborated with the American Board of Psychiatry and Neurology, Bristol-Myers Squibb, and the University of Virginia School of Medicine. PB-G has no personal conflicts of interest; her institution has received grants from Novartis, Beaufour Ipsen Pharma, Scor Global Life, Danone, and Lesieur. GF has no personal conflicts of interest; his institution has received a grant from the Alzheimer's Association. NCF has served on advisory boards for the Alzheimer's Research Forum and GE Healthcare. His institution has received remuneration, honoraria, and trial support from Abbott, the American Neurological Association, Lundbeck, GE Healthcare, GlaxoSmithKline, Elan Pharmaceuticals, Eli Lilly, Lundbeck, Eisai, IXICO, Pfizer, Janssen, the Movement Disorders Society, Wyeth Pharmaceuticals, Sanofi-Aventis, and Coalition Against Major Diseases Consortium as a result of his consultancy and advisory work. DG has served on the data safety monitoring board for a clinical trial for Jansen Pharmaceutical. GAJ has collaborated with Medivation, Martek Biosciences, and Avanir. JO'B has received honoraria for nonpromotional talks and travel/accommodation expenses from Pfizer, Eisai, Shire, Novartis, and Lundbeck, and his institute has undertaken consultancy work for GE Healthcare and Bayer Healthcare. FP has collaborated with Bayer Healthcare; her institution has received grants and honoraria from Servier, Bioprojet, Lundbeck, Eisai, Wyeth, Medivation, Exonhit Therapeutics, and Bristol-Myers Squibb. PR has collaborated with Lundbeck, Merck, Novartis, Janssen-Cilag, and Eisai. MR has served on a safety monitoring committee for Elan Pharmaceuticals, and is Director of the UK Dementia and Neurodegenerative Diseases Clinical Research Network. MS has collaborated with Eisai, Pfizer, Lundbeck, and Servier. LCdS has collaborated with Lundbeck. YS has collaborated with or consulted for Allergan, Cephalon, Elan, Eisai, Pfizer, Ortho-McNeil Neurologics, Merck \& Co, GlaxoSmithKline, Eli Lilly, and Janssen-Cilag. PJV has served on advisory boards for Bristol-Myers Squibb, Myriad, ElanWyeth, and Ipsen, and has consulted for Danone. His institution has received grants and honoraria from Bristol-Myers Squibb, Diagenic, Novartis, and Lundbeck. PS reports no personal conflict of interest; his institution has received grants from Wyeth, Elan, Genentech, Danone, Novartis, and Janssen-Cilag. CJ has participated in a scientific advisory board meeting organised by Bristol-Myers Squibb. AD, SG, HH, KM, and SS report no conflicts of interest. BD, HHF, NCF, and STDK have been involved in the working group of the American Association for the Revision of the NINCDS-ADRDA criteria for Alzheimer's disease.

\section{Acknowledgments}

The International Working Group received financial funding from Eisai France to support the meeting room cost for the ICAD 2009 meeting. The authors gratefully acknowledge discussion with Lon Schneider, University of Southern California, concerning the manuscript.

\section{References}

1 Cummings JL. Alzheimer's disease. N Engl J Med 2004; 351: 56-67.

2 Querfurth HW, LaFerla FM. Alzheimer's disease. N Engl J Med 2010; 362: 329-44.

3 McKhann G, Drachman D, Folstein M, Katzman R, Price D, Stadlan EM. Clinical diagnosis of Alzheimer's disease: report of the NINCDS-ADRDA Work Group under the auspices of Department of Health and Human Services Task Force on Alzheimer's Disease. Neurology 1984; 34: 939-44.

4 Bennett DA, Schneider JA, Arvanitakis Z, et al. Neuropathology of older persons without cognitive impairment from two community-based studies. Neurology 2006; 66: 1837-44.

5 Knopman DS, Parisi JE, Salviati A, et al. Neuropathology of cognitively normal elderly. J Neuropathol Exp Neurol 2003; 62: 1087-95.

6 Dubois B, Feldman HH, Jacova C, et al. Research criteria for the diagnosis of Alzheimer's disease: revising the NINCDS-ADRDA criteria. Lancet Neurol 2007; 6: 734-46.

7 Deramecourt V, Lebert F, Debachy B, et al. Prediction of pathology in primary progressive language and speech disorders. Neurology 2010; 74: 42-49.

8 Cummings J. Primary progressive aphasia and the growing role of biomarkers in neurological diagnosis. Ann Neurol 2008; 64: 361-64. 
9 Alladi S, Xuereb J, Bak T, et al. Focal cortical presentations of Alzheimer's disease. Brain 2007; 130: 2636-45.

10 Fagan AM, Mintun MA, Mach RH, et al. Inverse relation between in vivo amyloid imaging load and cerebrospinal fluid $A \beta_{42}$ in humans. Ann Neurol 2006; 59: 512-19.

11 Jagust WJ, Landau SM, Shaw LM, et al. Relationships between biomarkers in aging and dementia. Neurology 2009; 73: 1193-99.

12 Mintun MA, Larossa GN, Sheline YI, et al. $\left[{ }^{11} \mathrm{C}\right] \mathrm{PIB}$ in a nondemented population: potential antecedent marker of Alzheimer disease. Neurology 2006; 67: 446-52.

13 Buerger K, Ewers M, Pirttila T, et al. CSF phosphorylated tau protein correlates with neocortical neurofibrillary pathology in Alzheimer's disease. Brain 2006; 129: 3035-41.

14 Clark CM, Xie S, Chittams J, et al. Cerebrospinal fluid tau and beta-amyloid: how well do these biomarkers reflect autopsyconfirmed dementia diagnoses? Arch Neurol 2003; 60: 1696-702.

15 Ikonomovic MD, Klunk WE, Abrahamson EE, et al. Post-mortem correlates of in vivo PiB-PET amyloid imaging in a typical case of Alzheimer's disease. Brain 2008; 131: 1630-45.

16 Strozyk D, Blennow K, White LR, Launer LJ. CSF A $\beta 42$ levels correlate with amyloid-neuropathology in a population-based autopsy study. Neurology 2003; 60: 652-56.

17 Tapiola T, Alafuzoff I, Herukka SK, et al. Cerebrospinal fluid $\beta$-amyloid 42 and tau proteins as biomarkers of Alzheimer-type pathologic changes in the brain. Arch Neurol 2009; 66: 382-89.

18 National Institute on Aging, Reagan Institute Working Group on Diagnostic Criteria for the Neuropathological Assessment of Alzheimer's Disease. Consensus recommendations for the postmortem diagnosis of Alzheimer's disease. Neurobiol Aging 1997; 18 (4 suppl): S1-2.

19 Blennow K, Hampel H, Weiner M, Zetterberg H. Cerebrospina fluid and plasma biomarkers in Alzheimer disease. Nat Rev Neurol 2010; 6: 131-44.

20 Hampel H, Burger K, Pruessner JC, et al. Correlation of cerebrospinal fluid levels of tau protein phosphorylated at threonine 231 with rates of hippocampal atrophy in Alzheimer disease. Arch Neurol 2005; 62: 770-73.

21 Mattsson N, Zetterberg $\mathrm{H}$, Hansson $\mathrm{O}$, et al. CSF biomarkers and incipient Alzheimer disease in patients with mild cognitive impairment. JAMA 2009; 302: 385-93.

22 Visser PJ, Verhey F, Knol DL, et al. Prevalence and prognostic value of CSF markers of Alzheimer's disease pathology in patients with subjective cognitive impairment or mild cognitive impairment in the DESCRIPA study: a prospective cohort study. Lancet Neurol 2009; 8: 619-27.

23 Klunk WE, Engler H, Nordberg A, et al. Imaging brain amyloid in Alzheimer's disease with Pittsburgh Compound-B. Ann Neurol 2004; 55: 306-19.

24 Bourgeat P, Chetelat G, Villemagne VL, et al. Beta-amyloid burden in the temporal neocortex is related to hippocampal atrophy in elderly subjects without dementia. Neurology 2010; 74: 121-27.

25 Rowe CC, Ackerman U, Browne W, et al. Imaging of amyloid beta in Alzheimer's disease with 18F-BAY94-9172, a novel PET tracer: proof of mechanism. Lancet Neurol 2008; 7: 129-35.

26 Choi SR, Golding G, Zhuang Z, et al. Preclinical properties of 18F-AV-45: a PET agent for A $\beta$ plaques in the brain. J Nucl Med 2009; 50: 1887-94.

27 Li G, Sokal I, Quinn JF, et al. CSF tau $/ \mathrm{A} \beta_{42}$ ratio for increased risk of mild cognitive impairment: a follow-up study. Neurology 2007; 69: 631-39.

28 Ewers M, Buerger K, Teipel SJ, et al. Multicenter assessment of CSF-phosphorylated tau for the prediction of conversion of MCI. Neurology 2007; 69: 2205-12.

29 Hansson O, Zetterberg H, Buchhave P, Londos E, Blennow K, Minthon L. Association between CSF biomarkers and incipient Alzheimer's disease in patients with mild cognitive impairment: a follow-up study. Lancet Neurol 2006; 5: 228-34.

30 Henneman WJ, Vrenken H, Barnes J, et al. Baseline CSF p-tau levels independently predict progression of hippocampal atrophy in Alzheimer disease. Neurology 2009; 73: 935-40.

31 Morris JC, Roe CM, Grant EA, et al. Pittsburgh compound B imaging and prediction of progression from cognitive normality to symptomatic Alzheimer disease. Arch Neurol 2009; 66: 1469-75.
32 Resnick SM, Sojkova J, Zhou Y, et al. Longitudinal cognitive decline is associated with fibrillar amyloid-beta measured by $\left[{ }^{11} \mathrm{C}\right] \mathrm{PiB}$. Neurology 2010; 74: 807-15.

33 Teipel SJ, Ewers M, Wolf S, et al. Multicentre variability of MRI-based medial temporal lobe volumetry in Alzheimer's disease. Psychiatry Res 2010; 182: 244-50.

34 de Leon MJ, DeSanti S, Zinkowski R, et al. Longitudinal CSF and MRI biomarkers improve the diagnosis of mild cognitive impairment. Neurobiol Aging 2006; 27: 394-401.

35 Hampel H, Burger K, Teipel SJ, Bokde AL, Zetterberg H, Blennow K. Core candidate neurochemical and imaging biomarkers of Alzheimer's disease. Alzheimers Dement 2008; 4: 38-48.

36 Holland D, Brewer JB, Hagler DJ, et al. Subregional neuroanatomical change as a biomarker for Alzheimer's disease. Proc Natl Acad Sci USA 2009; 106: 20954-59.

37 Vemuri P, Whitwell JL, Kantarci K, et al. Antemortem MRI based STructural Abnormality iNDex (STAND)-scores correlate with postmortem Braak neurofibrillary tangle stage. Neuroimage 2008; 42: 559-67.

38 Patwardhan MB, McCrory DC, Matchar DB, Samsa GP Rutschmann OT. Alzheimer disease: operating characteristics of PET-a meta-analysis. Radiology 2004; 231: 73-80.

39 Whitwell JL, Josephs KA, Murray ME, et al. MRI correlates of neurofibrillary tangle pathology at autopsy: a voxel-based morphometry study. Neurology 2008; 71: 743-49.

40 Apostolova LG, Dutton RA, Dinov ID, et al. Conversion of mild cognitive impairment to Alzheimer disease predicted by hippocampal atrophy maps. Arch Neurol 2006; 63: 693-99.

41 de Leon MJ, Mosconi L, Blennow K, et al. Imaging and CSF studies in the preclinical diagnosis of Alzheimer's disease. Ann NY Acad Sci 2007; 1097: 114-45.

42 Devanand DP, Pradhaban G, Liu X, et al. Hippocampal and entorhinal atrophy in mild cognitive impairment: prediction of Alzheimer disease. Neurology 2007; 68: 828-36.

43 Okello A, Koivunen J, Edison P, et al. Conversion of amyloid positive and negative MCI to AD over 3 years: an ${ }^{11} \mathrm{C}$-PIB PET study. Neurology 2009; 73: 754-60.

44 Wolk DA, Price JC, Saxton JA, et al. Amyloid imaging in mild cognitive impairment subtypes. Ann Neurol 2009; 65: 557-68.

45 Jack CR Jr, Dickson DW, Parisi JE, et al. Antemortem MRI findings correlate with hippocampal neuropathology in typical aging and dementia. Neurology 2002; 58: 750-57.

46 Nordberg A, Rinne JO, Kadir A, Langstrom B. The use of PET in Alzheimer disease. Nat Rev Neurol 2010; 6: 78-87.

47 Barkhof F, Polvikoski TM, van Straaten EC, et al. The significance of medial temporal lobe atrophy: a postmortem MRI study in the very old. Neurology 2007; 69: 1521-27.

48 Braak H, Braak E, Bohl J, Reintjes R. Age, neurofibrillary changes, A $\beta$-amyloid and the onset of Alzheimer's disease. Neurosci Lett 1996 210: 87-90.

49 Jack CR Jr, Lowe VJ, Weigand SD, et al. Serial PIB and MRI in normal, mild cognitive impairment and Alzheimer's disease: implications for sequence of pathological events in Alzheimer's disease. Brain 2009; 132: 1355-65.

50 Mormino EC, Kluth JT, Madison CM, et al. Episodic memory loss is related to hippocampal-mediated beta-amyloid deposition in elderly subjects. Brain 2009; 132: 1310-23.

51 Mosconi L, Brys M, Switalski R, et al. Maternal family history of Alzheimer's disease predisposes to reduced brain glucose metabolism. Proc Natl Acad Sci USA 2007; 104: 19067-72.

52 Mosconi L, Mistur R, Switalski R, et al. Declining brain glucose metabolism in normal individuals with a maternal history of Alzheimer disease. Neurology 2009; 72: 513-20.

53 Reiman EM, Chen K, Alexander GE, et al. Correlations between apolipoprotein E $\varepsilon 4$ gene dose and brain-imaging measurements of regional hypometabolism. Proc Natl Acad Sci USA 2005; 102: 8299-302.

54 Reiman EM, Chen K, Alexander GE, et al. Functional brain abnormalities in young adults at genetic risk for late-onset Alzheimer's dementia. Proc Natl Acad Sci USA 2004; 101: 284-89.

55 Perrin RJ, Fagan AM, Holtzman DM. Multimodal techniques for diagnosis and prognosis of Alzheimer's disease. Nature 2009; 461: 916-22. 
56 Sarazin M, Berr C, De Rotrou J, et al. Amnestic syndrome of the medial temporal type identifies prodromal AD: a longitudinal study. Neurology 2007; 69: 1859-67.

57 Bouwman FH, Verwey NA, Klein M, et al. New research criteria for the diagnosis of Alzheimer's disease applied in a memory clinic population. Dement Geriatr Cogn Disord 2010; 30: 1-7.

58 Schoonenboom NS, van der Flier WM, Blankenstein MA, et al. CSF and MRI markers independently contribute to the diagnosis of Alzheimer's disease. Neurobiol Aging 2008; 29: 669-75.

59 de Jager CA, Honey TE, Birks J, Wilcock GK. Retrospective evaluation of revised criteria for the diagnosis of Alzheimer's disease using a cohort with post-mortem diagnosis. Int J Geriatr Psychiatry 2010; 25: 988-97.

60 Frisoni GB, Galluzzi S, Signorini M, et al. Preliminary evidence of validity of the revised criteria for Alzheimer disease diagnosis: report of 2 cases. Alzheimer Dis Assoc Disord 2010; 24: 108-14.

61 Aizenstein HJ, Nebes RD, Saxton JA, et al. Frequent amyloid deposition without significant cognitive impairment among the elderly. Arch Neurol 2008; 65: 1509-17.

62 Price JL, Morris JC. Tangles and plaques in nondemented aging and "preclinical" Alzheimer's disease. Ann Neurol 1999; 45: 358-68.

63 Galvin JE, Powlishta KK, Wilkins K, et al. Predictors of preclinical Alzheimer disease and dementia: a clinicopathologic study. Arch Neurol 2005; 62: 758-65.

64 Pike KE, Savage G, Villemagne VL, et al. Beta-amyloid imaging and memory in non-demented individuals: evidence for preclinical Alzheimer's disease. Brain 2007; 130: 2837-44.

65 Fagan AM, Roe CM, Xiong C, Mintun MA, Morris JC, Holtzman DM. Cerebrospinal fluid tau $/ \beta$-amyloid $_{42}$ ratio as a prediction of cognitive decline in nondemented older adults. Arch Neurol 2007; 64: 343-49.

66 Skoog I, Davidsson P, Aevarsson O, Vanderstichele H, Vanmechelen E, Blennow K. Cerebrospinal fluid beta-amyloid 42 is reduced before the onset of sporadic dementia: a populationbased study in 85-year-olds. Dement Geriatr Cogn Disord 2003; 15: 169-76.

67 Stomrud E, Hansson O, Blennow K, Minthon L, Londos E. Cerebrospinal fluid biomarkers predict decline in subjective cognitive function over 3 years in healthy elderly. Dement Geriatr Cogn Disord 2007; 24: 118-24.

68 Amieva H, Jacqmin-Gadda H, Orgogozo JM, et al. The 9 year cognitive decline before dementia of the Alzheimer type: a prospective population-based study. Brain 2005; 128: 1093-101.

69 Johnson DK, Storandt M, Morris JC, Galvin JE. Longitudinal study of the transition from healthy aging to Alzheimer disease. Arch Neurol 2009; 66: 1254-59.

70 Scahill RI, Schott JM, Stevens JM, Rossor MN, Fox NC. Mapping the evolution of regional atrophy in Alzheimer's disease: unbiased analysis of fluid-registered serial MRI. Proc Natl Acad Sci USA 2002; 99: 4703-07.

71 Cummings JL, Doody R, Clark C. Disease-modifying therapies for Alzheimer disease: challenges to early intervention. Neurology 2007; 69: $1622-34$

72 Dubois B. 'Prodromal Alzheimer's disease: a more useful concept than mild cognitive impairment? Curr Opin Neurol 2000; 13: 367-69.

73 Dubois B, Albert ML. Amnestic MCI or prodromal Alzheimer's disease? Lancet Neurol 2004; 3: 246-48.

74 Petersen RC, Smith GE, Waring SC, Ivnik RJ, Tangalos EG, Kokmen E. Mild cognitive impairment: clinical characterization and outcome. Arch Neurol 1999; 56: 303-08.
75 Ferri CP, Prince M, Brayne C, et al. Global prevalence of dementia: a Delphi consensus study. Lancet 2005; 366: 2112-17.

76 Barberger-Gateau P, Fabrigoule C, Helmer C, Rouch I, Dartigues JF. Functional impairment in instrumental activities of daily living: an early clinical sign of dementia? J Am Geriatr Soc 1999; 47: 456-62.

77 Braak H, Braak E. Neuropathological stageing of Alzheimer-related changes. Acta Neuropathol 1991; 82: 239-59.

78 Delacourte A, David JP, Sergeant N, et al. The biochemical pathway of neurofibrillary degeneration in aging and Alzheimer's disease. Neurology 1999; 52: 1158-65.

79 Price BH, Gurvit H, Weintraub S, Geula C, Leimkuhler E, Mesulam M. Neuropsychological patterns and language deficits in 20 consecutive cases of autopsy-confirmed Alzheimer's disease. Arch Neurol 1993; 50: 931-37.

80 Grossman M, Libon DJ, Forman MS, et al. Distinct antemortem profiles in patients with pathologically defined frontotemporal dementia. Arch Neurol 2007; 64: 1601-09.

81 Taylor KI, Probst A, Miserez AR, Monsch AU, Tolnay M. Clinical course of neuropathologically confirmed frontal-variant Alzheimer's disease. Nat Clin Pract Neurol 2008; 4: 226-32.

82 Khachaturian ZS. Diagnosis of Alzheimer's disease. Arch Neurol 1985; 42: 1097-105.

83 Frisoni GB, Lorenzi M, Caroli A, Kemppainen N, Nagren K, Rinne JO. In vivo mapping of amyloid toxicity in Alzheimer disease. Neurology 2009; 72: 1504-11.

84 Galton CJ, Patterson K, Xuereb JH, Hodges JR. Atypical and typical presentations of Alzheimer's disease: a clinical, neuropsychological neuroimaging and pathological study of 13 cases. Brain 2000; 123: 484-98.

85 Parnetti L, Tiraboschi P, Lanari A, et al. Cerebrospinal fluid biomarkers in Parkinson's disease with dementia and dementia with Lewy bodies. Biol Psychiatry 2008; 64: 850-55.

86 de Jong D, Jansen RW, Pijnenburg YA, et al. CSF neurofilament proteins in the differential diagnosis of dementia. J Neurol Neurosurg Psychiatry 2007; 78: 936-38.

87 Rowe CC, Ng S, Ackermann U, et al. Imaging beta-amyloid burden in aging and dementia. Neurology 2007; 68: 1718-25.

88 Gomperts SN, Rentz DM, Moran E, et al. Imaging amyloid deposition in Lewy body diseases. Neurology 2008; 71: 903-10.

89 McKeith IG, Dickson DW, Lowe J, et al. Diagnosis and management of dementia with Lewy bodies: third report of the DLB Consortium. Neurology 2005; 65: 1863-72.

90 Walker RW, Walker Z. Dopamine transporter single photon emission computerized tomography in the diagnosis of dementia with Lewy bodies. Mov Disord 2009; 24 (suppl 2): S754-59.

91 Walker Z, Jaros E, Walker RW, et al. Dementia with Lewy bodies: a comparison of clinical diagnosis, FP-CIT single photon emission computed tomography imaging and autopsy. J Neurol Neurosurg Psychiatry 2007; 78: 1176-81.

92 McKeith I, O'Brien J, Walker Z, et al. Sensitivity and specificity of dopamine transporter imaging with ${ }^{123} \mathrm{I}$-FP-CIT SPECT in dementia with Lewy bodies: a phase III, multicentre study. Lancet Neurol 2007; 6: 305-13.

93 Schneider JA, Aggarwal NT, Barnes L, Boyle P, Bennett DA. The neuropathology of older persons with and without dementia from community versus clinic cohorts. J Alzheimers Dis 2009; 18: 691-701. 